\title{
INTERAÇÃO ENTRE LEPTINA, GINÁSTICA ARTÍSTICA, PUBERDADE E EXERCÍCIO EM ATLETAS DO SEXO FEMININO*
}

\author{
MNDA. TATIANA DE BARROS MEIRA \\ Mestranda em estudos do esporte pela Universidade de São Paulo (USP) (São Paulo - Brasil) \\ E-mail: tati_meira7@hotmail.com
}

DRA. MYRIAN NUNOMURA

Professora da Escola de Educação Física e Esporte da USP, campus Riberão Preto

(São Paulo - Brasil)

E-mail: mnunomra@usp.br

\begin{abstract}
RESUMO
Há evidências de que as atletas de ginástica artística (GA) apresentam desregulações no sistema reprodutório em relação à média populacional. A interação entre alguns fatores pode explicar esse fato: genética, estresse do exercício, baixa porcentagem de gordura corporal, má nutrição e ação da leptina. Por meio de uma revisão de literatura, discutimos resultados de pesquisas que relacionam a ação do hormônio leptina e desregulações no sistema reprodutório durante a puberdade em atletas de GA do sexo feminino. Podemos afirmar que existe uma interação entre a seleção natural na modalidade, a baixa ingestão calórica e o estresse do treinamento, que ocasionaria a diminuição de leptina plasmática e causaria desregulações no sistema reprodutório durante a puberdade.
\end{abstract}

PALAVRAS-CHAVE: Ginástica artística; maturação, sistema reprodutório; leptina.

* O presente trabalho não contou com apoio financeiro de nenhuma natureza e tampouco houve conflitos de interesse para sua realização. 


\section{INTRODUÇÃO}

Há evidências de que as atletas de ginástica artística (GA) apresentam maturação tardia em relação à média populacional, ou seja, por volta dos 15 anos de idade (Malina et al., 2004; FerREIRA FILHO, 2007). No entanto, não existe um único fator determinante para esse fenômeno, podendo ser explicado pela interação de alguns fatores que atuam, conjuntamente, para esse resultado, entre os quais citamos o estresse causado pelo exercício, a herança genética, a baixa porcentagem de gordura corporal e o estado de má nutrição (quadro nutricional debilitado).

O treinamento de GA inicia-se cedo, por volta dos 6 anos de idade, e o pico de rendimento ocorre por volta dos 15 - 16 anos (NunOMURA et al., 2009). Para potencializar os resultados, as cargas de treinos aplicadas às crianças são altas e aquelas que apresentam estatura e massa menores e baixa taxa de gordura corporal são favorecidas para os movimentos técnicos da GA nos diversos aparelhos, o que pode gerar um estado nutricional debilitado a fim de buscar o perfil ideal para a modalidade.

Pesquisas recentes vêm demonstrando que a interação existente entre todos esses fatores pode estar associada à ação do hormônio leptina, produzido pelo tecido adiposo. Este tem relação direta com o sistema reprodutório e o processo da puberdade, bem como ação diferenciada quando as meninas são praticantes de exercícios físicos (BLum et al., 1997; WeIMANn et al., 1999; HORLICK et al., 2000; RoEmminch et al., 2002; Moschos et al., 2002; RoWLAND, 2005; Venner et al., 2006; RIBEIRO, 2007).

Assim, é importante conhecer a ação da leptina em atletas de GA para compreender como o treinamento pode interferir nas taxas desse hormônio e quais serão as consequências para as atletas durante a puberdade.

O objetivo desta revisão é discutir resultados de pesquisas recentes que demonstram a influência da leptina no processo pubertário de atletas de GA do sexo feminino, e as desregulações no sistema reprodutório que essas atletas possuem, apresentando-se uma visão contextualizada do tema, pois estudos nesse campo ainda são bastante recentes (WEIMANN et al., 1999; MuÑoz et al., 2004; ROWLAND, 2005; Fenichel; WarRen, 2007).

Para a realização da revisão de literatura, foram realizadas buscas em biblioteca, sites de busca (Pubmed, Medline e Scopus) e revistas especializadas nos diferentes temas: nutrição, treinamento esportivo, endocrinologia, medicina do esporte, metabolismo, pediatria e fisiologia, utilizando termos para busca: puberdade, leptina, tecido adiposo e amenorreia, ginastas e atletas, nos idiomas português e inglês. Para selecionar os artigos originais apresentados na revisão, consideramos a especificidade dos periódicos, levando em consideração os temas levantados e a data de 1990. 
De acordo com Ferreira Filho (2007), a GA é uma modalidade que reúne tanto elementos de força e leveza, que são demonstrados por movimentos graciosos e artísticos, como também saltos e acrobacias de alto grau de dificuldade.

Para atingir resultados expressivos na GA, além das capacidades físico-motoras e habilidades técnicas, as atletas devem apresentar características antropométricas específicas, como a baixa estatura, a baixa massa corporal e grande força muscular (Munôz et al., 2004; CLAesSEns et al., 2006). Os treinamentos na GA são iniciados em idades entre 5 e 7 anos (GEORgopoulos et al., 2004; ERLANDSON et al., 2008). Além disso, os praticantes/atletas dedicam muitas horas de treinamento por semana (entre 25 e 30 horas/semana) (BAXTER-Jones et al., 1994; GEORGOPOUlos et al., 2004; Theodoroupoulou et al., 2005; ERLANDSOn et al., 2008; NunOMURA et al., 2009).

Quando se analisa o perfil antropométrico de atletas da GA, verifica-se que estas apresentam baixa estatura, baixo peso corporal e baixas taxas de gordura corporal em relação à média populacional, tal como observado por Claessens et al. ( I 992), quando comparou peso e estatura de atletas de GA com o grupo controle da mesma idade cronológica (Tabela I).

Tabela I - Comparação entre médias de massa corporal e estatura entre ginastas e grupo controle

\begin{tabular}{|c|c|c|c|c|}
\hline & \multicolumn{2}{|c|}{ Massa corporal $(\mathrm{kg})$} & \multicolumn{2}{c|}{ Estatura (cm) } \\
\hline Idade (anos) & Ginastas & Controle & Ginastas & Controle \\
\hline $13+$ & $37,4 \pm 7,2$ & 49,0 & $143,3 \pm 9,4$ & 159,0 \\
\hline $14+$ & $42,8 \pm 6,0$ & 51,2 & $151,6 \pm 5,4$ & 161,7 \\
\hline $15+$ & $44,5 \pm 5,6$ & 53,8 & $154,0 \pm 6,3$ & 163,0 \\
\hline $16+$ & $47,5 \pm 5,6$ & 54,6 & $156,3 \pm 6,1$ & 163,4 \\
\hline $17+$ & $48,5 \pm 6,1$ & 56,2 & $156,8 \pm 6,2$ & 164,0 \\
\hline $18+$ & $47,8 \pm 6,1$ & 56,4 & $156,4 \pm 4,9$ & 164,2 \\
\hline
\end{tabular}

Fonte: Adaptado de Claessens et al. (1992).

No entanto, não é apenas o treinamento de GA que influencia nessas diferenças antropométricas, mas também o fator seleção natural. Isso porque se acredita que as meninas que apresentam essas características tendem a procurar a modalidade. Da mesma forma, aquelas que apresentam essas características se adequariam à modalidade e permaneceriam na prática e poderiam alcançar níveis elevados de rendimento (MaLINA et al., 2004). Conforme Nunomura e Tsukamoto (2006), as características como baixa estatura, baixo peso corporal, grande força 
muscular e proporcionalidade corporal favorecem biomecanicamente a execução de movimentos da GA.

Outro fator que evidencia a ocorrência da seleção natural na modalidade pode ser observado quando se avalia a idade de menarca de mães de atletas e se constata que a menarca ocorreu tardiamente. Segundo um estudo longitudinal, que comparou a idade de menarca de mães de ginastas, tenistas e nadadoras, verificou-se que, além de possuírem idade de menarca atrasada em relação à média populacional ( I 4,5 anos), as mães de ginastas apresentavam idade de menarca maior que aquela das mães de tenistas e de nadadoras. Além disso, a correlação entre a idade de menarca das ginastas e a das respectivas mães foi de $r=0,2$, e considerada moderada pelo autor, pois o máximo seria $r=0,5$, pelo fato de as filhas possuírem apenas metade dos genes em comum (ERLANDSON et al., 2008).

Em relação ao número de horas de treino semanais, percebe-se que esse valor é bastante expressivo. Comparando-se esse número com o número de horas de treino aos quais as ginastas eram expostas nas décadas de 1970 e 1980 ( 5 horas/semanais) e ao total que ocorre atualmente, verifica-se um aumento de praticamente 100\%. E, atualmente, esse número está por volta de 26 a 28 horas semanais (Georgopoulos et al., 2004; TheOdoroupoulou et al., 2005).

No Brasil, um estudo realizado por Nunomura et al. (2009) comparou a duração e a frequência do treinamento de GA em diferentes entidades competitivas do Brasil. E verificou-se que não existe um equilíbrio entre elas. E mais, o número de horas dedicadas aos treinos entre as crianças e os adolescentes equivalia ao treinamento de adultos, conforme a tabela 2.

Tabela 2 - Duração e frequência semanal de treino de atletas brasileiras de ginástica artística do sexo feminino

\begin{tabular}{|l|c|c|c|c|c|c|}
\hline $\begin{array}{c}\text { Categorias } \\
\text { competitivas }\end{array}$ & \multicolumn{2}{|c|}{$\begin{array}{c}\text { Duração da sessão } \\
\text { (horas) }\end{array}$} & \multicolumn{2}{c|}{$\begin{array}{c}\text { Frequência semanal } \\
\text { (dias/semana) }\end{array}$} & \multicolumn{2}{c|}{$\begin{array}{c}\text { Duração semanal } \\
\text { (horas) }\end{array}$} \\
\hline & $\begin{array}{c}\text { mínimo/ } \\
\text { máximo }\end{array}$ & $\begin{array}{c}\text { Média } \\
\pm \mathrm{dp}\end{array}$ & $\begin{array}{c}\text { mínimo/ } \\
\text { máximo }\end{array}$ & $\begin{array}{c}\text { Média } \\
\pm \mathrm{dp}\end{array}$ & $\begin{array}{c}\text { mínimo/ } \\
\text { máximo }\end{array}$ & $\begin{array}{c}\text { Média } \\
\pm \mathrm{dp}\end{array}$ \\
\hline Pré-infantil & $\mathrm{I}$ a 4 & $2,7 \pm 0,9$ & 2 a 6 & $3,4 \pm 1,5$ & 2 a 24 & $12 \pm 7$ \\
\hline Infantil & 3 a 6,5 & $3,8 \pm 0,91$ & 2 a 6 & $4,9 \pm 1,1$ & 6 a 32 & $18,9 \pm 7,9$ \\
\hline Juvenil & 3 a 6,5 & $4,7 \pm 1,2$ & 4 a 6 & $5,5 \pm 0,7$ & 12 a 32 & $24,0 \pm 5,1$ \\
\hline
\end{tabular}

Fonte: Adaptado de Nunomura et al. (2009).

As idades competitivas são definidas, no âmbito nacional, pela Confederação Brasileira de Ginástica (CBG) e, internacionalmente, pela Federação Internacional de Ginástica (FIG). A FIG estabeleceu que a idade mínima para participação nas competições internacionais é de 16 anos, a serem completados no ano da com- 
petição. Na tabela 3, estão apresentadas as categorias estabelecidas pela CBG para o setor feminino.

Tabela 3 - Categorias brasileiras femininas de ginástica artística

\begin{tabular}{|l|l|}
\hline \multicolumn{1}{|c|}{ Categorias } & \multicolumn{1}{c|}{ Idades } \\
\hline Pré-infantil & $09-10$ anos, completos no ano da competição \\
\hline Infantil & $10-12$ anos, completos no ano da competição \\
\hline Juvenil & $13-15$ anos, completos no ano da competição \\
\hline Adulto & A partir dos 13 anos \\
\hline
\end{tabular}

Fonte: Adaptado de CBG (2009).

\section{PUBERDADE, IDADE DE MENARCA E AMENORREIA EM ATLETAS DE GINÁSTICA ARTÍSTICA}

A puberdade é definida como um processo fisiológico de maturação hormonal e crescimento somático, que torna o organismo apto a se reproduzir (BARBOSA et al., 2006). Esse processo ocorre em todas as meninas, mas o tempo e a magnitude de cada fase ou evento podem diferir de uma para outra, ainda que, para a grande maioria, siga a mesma sequência de fatos (FALKNER; TANNER, 1986).

As principais manifestações e eventos que fazem parte do processo de puberdade no sexo feminino são o estirão de crescimento, a ativação funcional do sistema neuroendócrino (desenvolvimento de gônadas), o desenvolvimento de características sexuais secundárias femininas, a mudança na composição corporal (aumento de massa corporal, distribuição da gordura corporal, mudanças no índice de massa corporal) e a menarca (FAlKNeR; TANNER, 1986; BARBOSA et al., 2006)

As modificações hormonais são as primeiras a ocorrerem em relação às demais mudanças morfológicas. Essas modificações dizem respeito ao eixo hipotálamohipófise-gonadal (HPG), assim como seus respectivos hormônios. O funcionamento desse eixo ocorre desde o início da vida e é mantido estável por meio de mecanismos regulatórios, sendo reativado durante a puberdade (CHIPKEVITCH, 1995).

A partir das alterações das concentrações hormonais ocorridas antes e durante a puberdade, iniciam-se mudanças morfológicas muito importantes nas meninas. Essas mudanças morfológicas são conhecidas como características sexuais secundárias, e aquelas mais utilizadas em estudos com meninas são o desenvolvimento de mamas, o aparecimento de pelos pubianos e a idade de menarca (CHIPKEVITCH, 1995; MaLINA et al., 2004).

E mais, durante a puberdade ocorre o aumento de tecido adiposo, que leva ao aumento de massa corporal, mudança do índice de massa corporal (IMC), na porcentagem de gordura corporal e distribuição do tecido adiposo em determina- 
das partes do corpo feminino (RowLAND, 2005). O ganho total de tecido adiposo durante a puberdade em meninas gira em torno de $7,1 \mathrm{~kg}$ e a porcentagem de gordura aumenta em $21 \%$ aos $\mathrm{I}$ I anos de idade. $\bigcirc$ aumento de tecido adiposo nesse período etário provém da hipertrofia e da hiperplasia de células adiposas (FalkNeR; TANneR, 1986; Rowland, 2005).

A menarca é considerada o evento final do processo de maturação sexual e, também, muitas vezes o indicador de maturidade feminina (MALINA et al., 2004).

Há uma tendência mundial da idade da menarca ocorrer por volta dos 13 anos, mas, devido à heterogeneidade entre os países, sobretudo a situação econômica, esta pode variar significativamente. Falkner e Tanner (1986) afirmam que, em diversos países avaliados, a idade de menarca aumenta conforme a classe social diminui.

A maturação sexual feminina sofre grande influência de fatores genéticos, mas pode ser influenciada também pelo ambiente. Exercícios físicos de alta intensidade, assim como um estado nutricional debilitado, podem retardar a idade de menarca. E, geralmente, é a situação que ocorre com atletas de GA, que necessitam manter baixo peso e gordura corporal, além de participar de grande quantidade de treinamento espeć́fico e competições anuais (MunÔz et al., 2004; THEOdOROPOULOU et al., 2005).

O estudo conduzido por Claessens et al. (2006) comparou a maturação em atletas-ginastas com um grupo controle por meio da ocorrência de menarca, associada à maturação esquelética. Os autores verificaram que as ginastas apresentam idades de menarca e maturação esquelética tardia em relação à média populacional, ou seja, entre 14 e 17 anos.

Tabela 4 - Distribuição relativa (\%) de categorias maturacionais entre ginastas (G) e grupo controle (GC)

\begin{tabular}{|l|l|l|l|l|l|l|l|l|}
\hline \multicolumn{1}{|c|}{ Idade cronológica } & \multicolumn{2}{c|}{$14+$} & \multicolumn{2}{c|}{$15+$} & \multicolumn{2}{c|}{$16+$} & \multicolumn{2}{c|}{$17+$} \\
\hline População & G & GC & G & GC & G & GC & G & GC \\
\hline Categoria Maturacional $(n)$ & 43 & 709 & 41 & 534 & 29 & 543 & 37 & 462 \\
\hline Pré-menarcadas & $63 \%$ & $14 \%$ & $59 \%$ & $4 \%$ & $28 \%$ & $1 \%$ & $16 \%$ & $0 \%$ \\
\hline $\begin{array}{l}\text { Pós-menarcadas, mas não } \\
\text { maturadas esqueleticamente }\end{array}$ & $23 \%$ & $77 \%$ & $19 \%$ & $66 \%$ & $34 \%$ & $48 \%$ & $24 \%$ & $26 \%$ \\
\hline $\begin{array}{l}\text { Pós-menarcadas e maturadas } \\
\text { esqueleticamente }\end{array}$ & $14 \%$ & $19 \%$ & $22 \%$ & $30 \%$ & $38 \%$ & $51 \%$ & $60 \%$ & $74 \%$ \\
\hline
\end{tabular}

Fonte: Adaptado de Claessens et al. (2006).

Rowland (2005) afirma que muitas pesquisas comparam meninas atletas com não atletas e são encontrados valores de atraso na idade de menarca para aquelas atletas, o que é denominado de amenorreia primária. No entanto, muitas vezes, a seleção natural em determinadas modalidades esportivas privilegia meninas com 
menor quantidade de gordura corporal total, maior estatura e quadris estreitos, fatores estes que influenciariam o atraso da menarca e independentemente da menina praticar alguma atividade física ou não.

A amenorreia primária é definida como um retardo na idade de menarca ou a ausência de ciclo menstrual consecutivo durante três ou mais meses. $\bigcirc$ quadro de amenorreia secundária pode ser evidenciado quando uma menina com 16 anos de idade apresenta caracteres sexuais secundários femininos, mas não teve a menarca ou sofre atrasos no ciclo menstrual (ACSM, 1999).

O exercício físico pode ser um dos fatores causadores da amenorreia secundária, pois o atraso na idade de menarca é observado em vários esportes, mas não em todos. A idade média de menarca observada em meninas atletas é de 14 anos, e esse fato não deve ser considerado anormal, pois 95\% das meninas atingem a menarca entre I I e I 5 anos (MALINA et al., 2004).

Em contrapartida, a amenorreia hipotalâmica pode ser definida como um desarranjo no sistema reprodutor feminino que afeta o eixo hipotálamo-hipófisegonadal. $\bigcirc$ fato diminui a secreção de hormônios gonadotróficos e, assim, interfere na ocorrência do ciclo menstrual normal. As causas da amenorreia hipotalâmica estão associadas, principalmente, ao déficit energético, ou seja, à baixa ingestão calórica e ao alto gasto energético, este último pode ser ocasionado pelo exercício físico (Mantzoros, 2005).

Ginastas e bailarinas apresentam, consistentemente, maturação tardia em relação à população normal (não atletas ou não ativas) e 50\% delas apresentam quadros de amenorreia hipotalâmica (ROWLAND, 2005). Esse fato é atribuído tanto à alta intensidade de treinamento e à seleção natural, como ao estado nutricional debilitado, sendo os índices de disfunções alimentares em atletas de GA consideravelmente elevados ( I 5\% sofrem de anorexia e 62\% de bulimia) (BAPTISTA et al., 1997).

As ciências médicas não consideram a ocorrência da amenorreia hipotalâmica um sintoma normal causado pelo exercício. Ao contrário, esta é entendida como uma disfunção séria, pois a reversão desse quadro não é atingida facilmente. Além disso, um quadro de amenorreia em atletas pode ser seguido de outros dois problemas graves como os distúrbios alimentares e a osteoporose, que caracterizam a chamada tríade da atleta (ACSM, 1999; STAFFORD, 2005).

\section{RELAÇÃO ENTRE LEPTINA E ATLETAS DE GINÁSTICA ARTÍSTICA}

A leptina é uma proteína composta de 146 aminoácidos de peso molecular de $16 \mathrm{kDa}$, que circula no sangue tanto na forma livre, como em conjunto com proteínas carregadoras. Ela possui estrutura terciária que se assemelha à classe de 
citocinas I (BLum et al., 1997; MANTZOros, 2005). Sua liberação ocorre em pulsos durante o dia, sendo que os maiores picos de secreção ocorrem a partir da meianoite, segue durante a madrugada e diminui no início da manhã.

A produção de leptina ocorre, predominantemente, no tecido adiposo branco, em maior quantidade no tecido subcutâneo comparado ao visceral e é produzida, também, em menores quantidades no estômago, placenta e glândulas mamárias (MANTZOROS, 2005; RiBEIRO et al., 2007).

A ação da leptina no hipotálamo ocorre, especificamente, no núcleo arqueado, estimulando e/ou inibindo os neurônios orexígenos e anorexígenos e controlando, assim, os mecanismos de fome e de saciedade e, também, os mecanismos de ingestão de alimentos e de gasto energético. Baixas concentrações de leptina plasmática levam ao aumento da ingestão alimentar, fome e diminuição do gasto energético, enquanto altas concentrações dela causam diminuição da ingestão alimentar, saciedade e aumento do gasto energético (RIBEIRO et al., 2007).

As oscilações do peso corporal regulam, indiretamente, as concentrações de leptina plasmática. A perda do peso corporal provoca a diminuição de leptina plasmática e o aumento, em contrapartida, provoca a elevação da concentração dela. Assim, as concentrações de leptina plasmática sinalizam para o sistema nervoso central (SNC), informando sobre as reservas de energia estocadas em forma de tecido adiposo (Negrão; Licinio, 2000; Mantzoros, 2005, Benatti; Lancha Junior, 2007).

Além da comunicação que a leptina tem com o hipotálamo, esta também possui receptores em outros órgãos e células, como, por exemplo, na glândula hipófise anterior, nas células gonadotróficas, nos ovários, nas células de Leyding e no endométrio. Esse fato demonstra o sistema complexo de regulação do eixo HPG por meio da ação do hormônio leptina durante o processo pubertário (Moschos et al., 2002).

\section{LEPTINA E PUBERDADE NO SEXO FEMININO}

A relação entre a menarca e o sistema reprodutório feminino com a leptina tem sido alvo de pesquisas nos últimos anos e estas demonstram que a leptina tem correlação positiva com medidas de composição corporal, como massa de gordura corporal, IMC, peso e medidas antropométricas em meninas. $\bigcirc$ aumento da massa de gordura corporal, assim como sua distribuição específica no corpo feminino, provoca a elevação das concentrações de leptina sanguínea (HORLICK et al., 2000; Lassek; Gaulin, 2007; Kulik-ReChberger et al., 2008).

Sabe-se que o aumento da concentração de leptina plasmática tem ação direta como hormônio regulatório do sistema reprodutório e, consequentemente, atua durante o processo pubertário. Sua ação é considerada de extrema importância 
durante ele, pois atua como um agente permissivo para o início da puberdade (Moschos et al., 2002).

Verificou-se que o aumento de leptina sanguínea estava inversamente correlacionado à idade de menarca, apresentando picos em torno de 12 anos, idade média de ocorrência de menarca. Pode-se explicar o fato, pois o aumento da concentração sanguínea de leptina é um dos primeiros sinais de iniciação e ativação do eixo HPG, e resulta no aumento das concentrações de hormônios esteroides (no caso do feminino: estrógeno e progesterona) (MATKOVIC et al., 1997).

Além disso, as pesquisas demonstraram o aumento progressivo das concentrações de leptina durante o processo pubertário (estágios I a 5 de Tanner) (BLum et al., 1997; HorLICK et al., 2000; KULIK-RECHBERGER et al., 2008), fato que comprova que existe relação direta desse hormônio durante todo o processo pubertário.

\section{AÇÃO DA LEPTINA EM ATLETAS DE GINÁSTICA ARTÍSTICA}

Pesquisas referentes às relações entre leptina e exercício concluíram que, quanto maior o nível de estresse causado pelo exercício, menores quantidades de leptina plasmática eram encontradas (LAUGHLIN; YeN, 1997; WeIMANn et al., 1999; PUder et al., 2006).

Conforme verificado por meio de revisão bibliográfica conduzida por Venner et al. (2006), programas de exercícios físicos tendem a gerar balanço energético negativo, perda de peso e perda de massa gorda, o que pode ocasionar diminuição da concentração de leptina plasmática.

Nas pesquisas em que a amostra foi composta por bailarinas e ginastas, foram observadas relações entre as baixas concentrações de leptina, os estados nutricionais debilitados e as medidas antropométricas abaixo da média (WEIMANN et al., 1999; MuÑoz et al., 2004). No caso na GA, esse fato pode estar relacionado à seleção natural na modalidade, que prioriza meninas com medidas antropométricas abaixo da média (Rowland, 2005), aos quadros de disfunções alimentares apresentadas por atletas dessa modalidade, assim como a exposição às altas cargas de treinamento desde a infância (BAPTISTA et al., 1997).

Mediante a observação dos resultados das pesquisas apresentadas, é possível afirmar que o balanço energético negativo (exercício associado a um estado nutricional debilitado) tem relação com as baixas concentrações de leptina plasmática e a desregulação do sistema reprodutório (LAUGHLIN; YEN, 1997; WEIMANN et al., 1999; THONG et al., 2000; Muñoz et al., 2004; KASSA-VuBu et al., 2006, Meira et al., 2009).

A cobrança pelo peso e gordura corporal em níveis baixos induz as ginastas a restringirem o consumo calórico com a intenção de manter ou mesmo diminuir esses índices. Um questionário aplicado entre ginastas alemães (sexo feminino e masculino) verificou que aqueles do masculino não fazem restrição alimentar. Em contrapartida, 
as atletas do sexo feminino afirmam que ingerem alimentos com baixas calorias e demonstram preocupação em conhecer sobre os diferentes nutrientes presentes na sua dieta diária (WEIMANN, 2002). Essas evidências corroboram com as conclusões de um estudo brasileiro em que atletas de GA com idades entre I | e I 4 anos foram entrevistadas, quando foi constatado que as ginastas consomem, em média, I.450 calorias/dia, enquanto o recomendado é de 2.200 calorias/dia (RibeIRO; SOARES, 2002).

Sugere-se que as concentrações de leptina poderiam influenciar a regulação do sistema ósseo, pois receptores de leptina são encontrados no tecido ósseo. Assim, quadros de osteopenia e osteoporose estariam relacionados à amenorreia e aos estados nutricionais debilitados (tríade da mulher atleta), e poderiam estar associados às concentrações de leptina. $\bigcirc$ fato foi verificado em um estudo que comparou as concentrações de leptina e a densidade óssea de ginastas e bailarinas com meninas ativas. Esse estudo concluiu que as ginastas possuem menor densidade óssea, idade de menarca atrasada e menores concentrações de letpina sanguínea (Munõz et al., 2004; Fenichel; WarRen, 2007).

Em relação aos estágios pubertários (estágios I a 5 de Tanner), em atletas de GA, observa-se que estas não apresentam relação com concentrações de leptina plasmática. Um estudo conduzido por Weimann (2002) verificou que as concentrações de leptina aumentam do estágio I para II, mas diminuem nos estágios III e IV e chegam a ficar menores em relação ao estágio I. Suspeita-se que, além de possuírem taxas menores de leptina durante a puberdade, as atletas de GA não possuem padrão de secreção de letpina relacionados aos estágios pubertários.

\section{CONSIDERAÇÕES FINAIS}

Em atletas de GA do sexo feminino, a queda da concentração de leptina plasmática pode estar associada ao balanço energético negativo. Este, por sua vez, pode ser causado pela diminuição do peso corporal, que pode ter relação com as baixas reservas de gordura e ingestão alimentar que, muitas vezes, é deficiente. Esse fato pode levar à inibição do eixo hipotálamo-hipófise-gonadal e à interrupção da função reprodutiva, provocando desregulações no sistema reprodutório, como atraso na idade de menarca e a amenorreia hipotalâmica.

Assim, podemos afirmar que existe uma interação entre a seleção natural na modalidade, a baixa ingestão calórica e o estresse causado pelo treinamento intensivo da GA, que ocasionaria a diminuição de leptina plasmática, o que desregula o sistema reprodutório durante a puberdade. E, como consequência, as ginastas poderiam apresentar idade de menarca atrasada, disfunções ósseas, amenorreia e tríade da mulher atleta. 
Estudos futuros poderiam investigar, longitudinalmente, a influência do treinamento de GA nas concentrações de leptina, na ocorrência de distúrbios alimentares e nos desarranjos do sistema reprodutório, a fim de encontrar a melhor forma de desenvolver o treinamento. E, assim, adequar o treinamento à duração e à intensidade de treino, idade de início de prática, indicações nutricionais e capacidades físicas a serem desenvolvidas em cada idade, e visar ao alto rendimento na modalidade e sem prejudicar o desenvolvimento das atletas.

O presente estudo indica que, na condução do treinamento de GA, os profissionais deveriam fazer o acompanhamento da idade de menarca (preferencialmente com a ajuda de médicos), aferir a idade de menarca das mães, orientar sobre os riscos da dieta desequilibrada, permitir períodos de descanso dos treinos e tempo de sono satisfatório. E, finalmente, adequar as cargas de treinos ao período pré-pubertário com a intenção de diminuir os efeitos nocivos do treinamento nessa fase sensível de vida.

Interaction between among leptin, artistic gymnastic, puberty and exercise in female athletes

ABSTRACT: There are evidences that artistic gymnastic (AG) athletes have late maturation compared to the average population. The interplay of some factors may explain it, such as: hereditary factors, exercise stress, low fat mass percentage, bad nutrition and the leptin hormone action. The aim of this literature review is to discuss the results of studies regarding the leptin hormone action and the deregulation in the reproductive system during the puberty of AG female athletes. It is possible to affirm that there is a relation between natural selection in the modality, low caloric diet and the training stress in the AG, resulting in the decrease of the level of the plasmatic leptin, which de-regulates the reproductive system during the puberty.

KEY WORDS: Artistic gymnastics; maturation, reproductive system, puberty; leptin.

\section{Interacción entre leptina, gimnastica artística, pubertad e ejercicio en atletas del sexo femenino}

RESUMEN: Hay evidencias de que las atletas de gimnasia artística (GA) presentan madurez tardía en relación a la media poblacional. La interacción entre algunos factores puede explicar este hecho, como: factores hereditarios, estrés del ejercicio, baja porcentaje de gordura corporal, mala nutrición y la acción de la hormona leptina. Por medio de una revisión de la literatura, discutiremos los resultados de estudios que relacionan la acción del la hormona leptina y las desregulaciones en el sistema reproductivo durante la pubertad en atletas de GA del sexo femenino. Es posible afirmar que existe una interacción entre la selección natural de la modalidad, una baja ingestión calórica y el estrés del entreno, que ocasionaría la disminución de la concentración de leptina plasmática y causaría desregulaciones en el sistema reproductivo durante la pubertad.

PALABRAS CLAVES: Gimnasia artística; maduración; sistema reproductivo; pubertad; leptina. 


\section{REFERÊNCIAS}

AMERICAN COLLEGE OF SPORTS MEDICINE - ACSM. Posicionamento oficial: a tríade da atleta. Revista Brasileira de Medicina do Esporte, v. 5, n. 3, 1999.

BAPTISTA, C.; GATTI, A. O; SUZUKI, M. E. S. et al. Ginástica olímpica: considerações nas atletas do Centro Olímpico de Treinamento e Pesquisa. Revista Âmbito Medicina Desporto, v. 6, p. 19-26, 1997.

BARBOSA, K. B. F.; FRANCESCHINI, S. C. C.; PRIORE, S. E. Influência dos estágios de maturação sexual no estado nutricional, antropometria e composição corporal de adolescentes. Revista Brasileira de Saúde Maternidade Infantil, v. 6, n. 4, p. 375-383, 2006.

BAXTER-JONES, A.D.G.; HELMS, P.; BAINES-PREECE, J.; PREECE, M. M. Menarche in intensively trained gymnasts, swimmers and tennis players. Annals of Human Biology, v. 21 , n. 5, p. 407-415, 1994.

BENATTI, F. B.; LANCHA JUNIOR, A. H. Leptina e exercício físico aeróbio: implicações da adiposidade corporal e insulina. Revista Brasileira de Medicina do Esporte, v. 13, n. 4, p. 263-269, 2007.

BLUM, W. F; ENGLANO, P.; HANITSCH, S. et al. Plasma leptin levels in healthy children and adolescents: dependence on body mass index, body fat mass, gender, pubertal stage, and testosterone. J Clinical Endocrinology \& Metabolism, v. 82, n. 9, p. 2.904-2.910, 1997.

CHIPKEVITCH, E. Puberdade \& adolescência: aspectos biológicos, clínicos e psicossociais. São Paulo: Rocco, 1995.

CLAESSENS, A. L.; MALINA, R. M.; LE FEVRE, J. et al. Growth and menarcheal status of elite female gymnasts. Medicine \& Science in Sports \& Exercise, v. 24, n. 7, p. 755-763, 1992.

. Maturity-associated variation in the body size and proportions of elite female gymnasts |4- 17 years of age. European Journal of Pediatrics, v. I65, n. 3, 2006.

CONFEDERAÇÃO BRASILEIRA DE GINÁSTICA - CBG. Ginástica artística feminina Regulamento técnico do campeonato nacional. [on-line] Disponível em: < http://cbginastica. com.br>. Acesso em: jun. 2009.

ERLANDSON, M. C.; SHERAR, L. B.; MIRWALD, R. L. et al. Growth and maturation of adolescent female gymnasts, swimmers, and tennis players. Medicine \& Science in Sports \& Exercise, v. 40, n. I, p. 34-42, 2008.

FALKNER, F;; TANNER, J. M. Human growth: a comprehensive treatise. New York: Plenum Press, 1986.

FENICHEL, R. M.; WARREN, M. P. Anorexia, bulimia, and the athletic triad: evaluation and management. Current Osteoporosis Reports, v. 5, n. 4, 2007. 
FEREIRA FILHO, R. A. Estudo sobre a estatura de ginastas na ginástica artística feminina de alto nível no Brasil. Dissertação (Mestrado) - Escola de Educação Física e Esporte da Universidade de São Paulo, 2007.

GEORGOPOUlOS, N. A.; THEODORO POULOU, A.; LEGLISE, M.; APOSTOLOS, G. V.; KOSTAS, B. M. et al. Growth and skeletal maturation in male and female artistic gymnasts. The Journal of Clinical Endocrinology \& Metabolism, v. 89, n. 9, p. 4.377-4.382, 2004.

HORLICK, M. B.; ROSENBAUM, M.; NICHOLSON, M. et al. Effect of puberty on the relationship between circulating leptin and body composition. The Journal of Clinical Endocrinology \& Metabolism, v. 85, n. 7, p. 2.509-2.518, 2000.

KASSA-VUBU JZ, Y. E. W.; YE, N.; BORER, T.; ROSENTHAL, A.; MECKMONGKOL, T. Twenty-four hour growth hormone and leptin secretion in active postpubertal adolescent girls: impact of fitness, fatness, and age at menarche. The Journal of Clinical Endocrinology \& Metabolism, v. 91, n. 10, p. 3.925-3.940, 2006.

KULIK-RECHBERGER, B.; TARKOWSKI, R.; MAGOFFIN, D. A. et al. Leptin gene expression in subcutaneous adipose tissue in girls before and during puberty. Eur J Obstetrics \& Gynecol Reprod Biol., v. 136, p. 210-214, 2008.

LASSEK, W. D.; GAULIN, S. J. C. Brief communication: menarche is related to fat distribution. American Journal of Physical Anthropology. v. 133, p. I. |47- I. I 51, 2007.

LAUGHLIN, G. A.; YEN, S. S. C. Hypoleptinemia in women athletes: absence of diurnal rhythm with amenorrhea. The Journal of Clinical Endocrinology \& Metabolism, v. 82, n. I, p. $318-32|| 997$.

MALINA, R. M.; BOUCHARD, C.; BOR-OR, O. Growth, maturation, and physical activity. Champaign Illinois: Human Kinetics, 2004.

MANTZOROS, J. L. C. C. Role of leptin in energy-deprivation states: normal human physiology and clinical implications for hypothalamic amenorrhea and anorexia nervosa. Lancet, v. 356, p. 74-85, 2005.

MATKOVIC, V.; ILICH, J. Z.; SKUGOR, M. et al. Leptin is inversely related to age at menarche in human females. The Journal of Clinical Endocrinology \& Metabolism, v. 82, n. I0, p. 3.239-3.244, 1997.

MEIRA, T. B.; MORAES, F. L.; BÖHME, M. T. S. Relações entre leptina, puberdade e exercício no sexo feminino. Revista Brasileira de Medicina do Esporte, v. I5, n. 4, p. 306-3 I0, 2009.

MOSCHOS, S.; CHAN, J. L.; MANTZOROS, M. D. Leptin and reproduction: a review. Fertility and Sterility, v. 77, n. 3, p. 433-444, 2002. 
MUÑOZ, M. T.; PIEDRA, C.; BARRIOS, V.; GARRIDO, G.; AREENTE, J. Changes in bone density and bone markers in rhythmic gymnasts and ballet dancers: implications for puberty and leptin levels. European Journal of Endocrinology, v. I 51, p. 491-496, 2004.

NEGRÃO, A. B.; LICINIO, J. Leptina: o diálogo entre adipócitos e neurônios. Arquivo Brasileiro de Endocrinologia e Metabolismo, v. 44, n. 3, p. 205-2। 4, 2000.

NUNOMURA, M.; PIRES, F. R.; CARRARA, P. Análise do treinamento na ginástica artística brasileira. Revista Brasileira de Ciências do Esporte, Campinas, v. 3I , n. I , p. 25-40, 2009.

:; TSUKAMOTO, M. H. C. Análise crítica da ginástica olímpica. In: TANI, G.; BENTO, J. O.; PETERSEN, R. D. S. Pedagogia do desporto. Rio de Janeiro: Guanabara-Koogan, 2006. p. 355-37I.

PUDER, J. J.; MONACO, S.E.; SEN GUPTA, S. et al. Estrogen and exercise may be related to body fat distribution and leptin in young women. Fertility and Sterility, v. 86, p. 694-699, 2006.

RIBEIRO, B. G.; SOARES, E. A. Avaliação do estado nutricional de atletas de ginástica olímpica do Rio de Janeiro e São Paulo. Revista de Nutrição, v. I5, n. 2, p. |8| - |9| , 2002.

RIBEIRO, S. M. L.; SANTOS, Z. A.; SILVA, R. J. et al. Leptina: aspectos sobre o balanço energético, exercício físico e amenorreia do esforço. Arquivo Brasileiro de Endocrinologia e Metabolismo, v. 5I, n. I, p. II-24, 2007.

ROEMMINCH, J. N.; CLARK, P.; WALTER, K. et al. Pubertal alterations in growth and body composition. VI. Pubertal insulin resistance: relation to adiposity, body fat distribution and hormone release. Int J Obes Relat Metab Disord., v. 26, p. 701-709, 2002.

ROWLAND, T. W. Children's exercise physiology. Champaign Illinois: Human Kinetics, 2005.

STAFFORD, D. E. J. Altered hypothalamic-pituitary-ovarian axis function in young female athletes implications and recommendations for management. Treatment and Endocrinology, v. 4, n. 3, p. 147-154, 2005.

THEODOROPOULOU, A.; MARKOU, K. B.; VAGENAKIS, G. A. et al. Delayed but normally progressed puberty is more pronounced in artistic compared with rhythmic elite gymnasts due to the intensity of training. The Journal of Clinical Endocrinology \& Metabolism, v. 90, n. I I, p. 6.022-6.027, 2005.

THONG, F. S. L.; MCLEAN, C.; GRAHAM, T. E. et al. Plasma leptin in female athletes: relationship with body fat, reproductive, nutritional, and endocrine factors. Journal of Applied Physiology, v. 88, p. 2.037-2.044, 2000.

VENNER, A. A.; LYON, M. E.; DOYLE-BAKER, P. K. Leptin: a potencial biomarker for childhood obesity? Clin Biochemistry, v. 39, p. I.047-1.056, 2006. 
WEIMANN, E. Gender-related differences in elite gymnasts: the female athlete triad. Journal of Applied Physiology, v. 92, p. 2.146-2.152, 2002.

; BLUM, W. F.; WITZEL, C.; SCHWIDERGALL, S.; BÖHLES, H. J. Hypoleptinemia in female and male elite gymnasts. European Journal of Clinical Investigation, v. 29, p. 853-860, 1999.

Recebido: 26 set. 2009

Aprovado: 31 mar. 2010

Endereço para correspondência:

Tatiana de Barros Meira

Estrada do Embu, I.563, casa B27S - Jardim Torino

Cotia-SP

CEP 067।3-100 